\title{
Analysis of Microspike Movements on the Neuronal Growth Cone ${ }^{1}$
}

\author{
D. BRAY ${ }^{2}$ AND K. CHAPMAN \\ Medical Research Council, Cell Biophysics Unit, 26 Drury Lane, London WC2B 5RL, United Kingdom
}

\begin{abstract}
Growth cones of chick sensory ganglion neurons in tissue culture were photographed at $60-\mathrm{sec}$ intervals as they advanced over the substratum. Numbers of microspikes (or "filopodia") were recorded together with the time and position of their appearance, their rate of elongation, their lateral movements, their lifespan, and the position and manner of their disappearance. All microsplkes go through cycles of extension, lateral movement, and shortening. These are irregular and unpredictable but show systematic differences depending on where on the the growth cone they occur. At the leading edge of the growth cone microspike extension occurs at highest frequency and microspike shortening occurs at the lowest frequency; when the latter occurs in this region it often involves the advance of the margin of the cell in the form of a lamellipodium. Microspike loss occurs most often at the base of the growth cone, usually by the retraction of the microspike into the cell. Calculations of the gain and loss of microspikes at different regions of the growth cone show that they undergo a net retrograde flow, the rate of which is correlated with the forward advance of the growth cone. Individual microspikes can also move backward from the growth cone onto the axon (or "neurite"), an event that occurs most often on adhesive substrata.
\end{abstract}

Our observations support a direct role of microspike movement in the advance of the growth cone. The primary force for axonal elongation appears to be the contraction of microspikes pulling the leading margin of the growth cone forward. At more proximal and peripheral regions of the growth cone, microspikes undergo a retrograde sweeping motion, followed by retraction into the cell, which may also contribute to the forward movement of the growth cone. We interpret these movements as arising from a flow of actin filaments and associated proteins which are incorporated into microspikes and lamellipodia at the leading edge of the growth cone, passing backward, and being deposited into the actin-rich membrane-associated cortex of the axonal cylinder.

Many qualitative descriptions of the neuronal growth cone have been published in the eighty or so years since its discovery (Bunge et al., 1983; Landis, 1983; Kater and Letourneau, 1985). Its form in tissue culture and in the developing embryo ranges from a simple bulbous club to an ornate efflorescence of surface protrusions, consisting mainly of hair-like microspikes (or "filopodia") and thin flat

Received February 14, 1985; Revised May 8, 1985;

Accepted May 13, 1985

\footnotetext{
'Many thanks to Mary Bunge, Julian Heath, Kathy Tosney, and the reviewers for their helpful comments on this work.

${ }^{2}$ To whom correspondence should be addressed.
}

veils of lamellipodia (Roberts and Taylor, 1983). Its movements, difficult to convey in words, comprise minute-by-minute extensions and retractions of microspikes and lamellipodia, superimposed on a more stately advance of the entire structure as the axon or dendrite grows. Although an axon may have some innate ability to elongate without conspicuous surface activity, under most conditions in tissue culture or in the animal, surface protrusions such as microspikes are essential for axonal growth and guidance (Wessells and Nuttall, 1978; Goodman and Bastiani, 1984; Marsh and Letourneau, 1984).

So far as we are aware, however, quantitative analyses of growth cones have so far been limited to their overall rates of advance (see, for example, Ludueña, 1973; Argiro et al., 1984; Katz et al., 1984), and the rate of elongation of their microspikes (Argiro et al., 1985). There has been no systematic analysis that would permit one to say, objectively, how a growth cone moves from point $A$ to point $B$, even on a planar culture substratum. Where and when on the growth cone are micruspikes and lamellipodia formed? Where and when are they lost and how frequently? Do they move like limbs or oars that drag the growth cone forward? Or are their formation and movement independent of the growth cone advance, indicating a more sensory role? Can microspikes that have made contact with a surface move again, and if so, do they appear to be anchored principally at their base or their tip? If they cannot move, once attached, what happens to them as the growth cone moves past? How are the lateral microspikes on the neurite formed: do they move backward from the growth cone or are they formed in situ? When and how do microspikes retract, and does this phenomenon play any role in growth cone locomotion? Questions such as these must be answered if we are to understand the locomotory and navigational capabilities of the growth cone, on which so much of early neuronal development depends.

\section{Materials and Methods}

Tissue culture. Cells were derived from the lumbar sensory ganglia of chicken embryos at the 10th to 12th day of incubation. The ganglia were removed aseptically and incubated for $25 \mathrm{~min}$ at $37^{\circ} \mathrm{C}$ in $0.25 \%$ trypsin in Hanks' balanced salt solution (HBSS) lacking $\mathrm{Ca}^{2+}$ and $\mathrm{Mg}^{2+}$ (Flow Laboratories, Irvine, Scotland). At the end of the incubation period, the trypsin was removed and the ganglia were washed several times in complete HBSS and then repeatedly forced through the tip of a Pasteur pipette. This resulted in the dissociation of the ganglia into single cells.

Aliquots of the cell suspension equivalent to half a ganglion (about 1500 neurons) were seeded into a sterile assembly consisting of a $1-\mathrm{cm}$ glass ring placed on a $22 \times 22 \mathrm{~mm}$ coverslip on the bottom of a $35-\mathrm{mm}$ plastic tissue culture dish. The dish also contained $3 \mathrm{ml}$ of Leibovitz L-15 medium (Flow Laboratories) supplemented with $10 \%$ fetal calf serum, $0.6 \%$ glucose, $2 \mathrm{~mm}$ L-glutamine, 100 units $/ \mathrm{ml}$ of penicillin, $100 \mu \mathrm{g} / \mathrm{ml}$ of streptomycin, $0.6 \%$ methylcellulose, and an amount of mouse nerve growth factor adequate to support a healthy outgrowth of neurites. Cells were kept in a humidified incubator at $37^{\circ} \mathrm{C}$ for 18 to $20 \mathrm{hr}$ before observation.

Adhesive substrata were prepared essentially as described by Collins (1978). A thin layer of carbon was shadowed onto glass coverslips followed by treatment with a solution of poly-DL-lysine (Sigma; $M_{r}=30,000$ to 70,000 , 

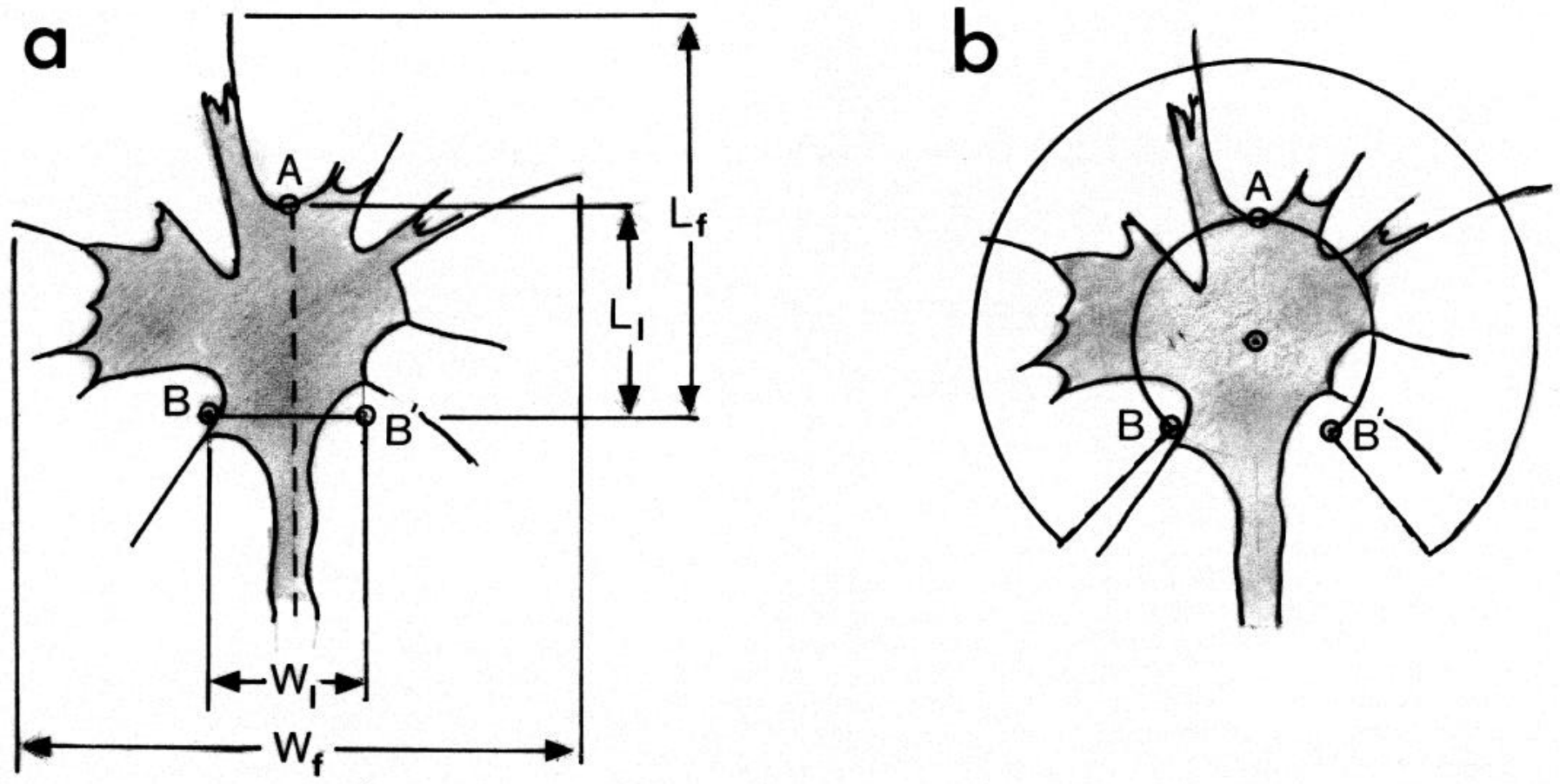

Figure 1. Measurements of growth cones. $a$, Dimensions described in the text. $b$, Circular arcs used to measure microspike spacing and rate of movement. The inner arc, drawn through points $A B B^{\prime}$, is taken as the locus of the microspike bases. The outer arc, representing the sweep of microspike tips, is concentric with the inner circle and has diameter, $W_{f}$.

TABLE I

Statistics of an average growth cone

The statistics are based on the examination of nine sensory growth cones in culture, as described in the text. Note that these were selected for their relatively small number of microspikes (see Materials and Methods). Data are given $\pm \mathrm{SEM}$, with the number of measurements indicated in parentheses.

\begin{tabular}{|c|c|}
\hline Characteristic & Measurement \\
\hline Overall length $\left(L_{f}\right.$ in Fig. 1$)$ & $18.16 \pm 1.39 \mu \mathrm{m}(45)$ \\
\hline Base length $\left(L_{l}\right.$ in Fig. 1$)$ & $9.58 \pm 0.73 \mu \mathrm{m}(54)$ \\
\hline Overall width $(W$, in Fig. 1$)$ & $28.11 \pm 1.37 \mu \mathrm{m}(45)$ \\
\hline Base width ( $W_{l}$ in Fig. 1) & $6.95 \pm 0.88 \mu \mathrm{m}(9)$ \\
\hline Growth velocity (see Fig. 2) & $1.08 \pm 0.26 \mu \mathrm{m} / \mathrm{min}(9)$ \\
\hline No. of microspikes (per growth cone) & $9.22 \pm 0.57(9)$ \\
\hline Microspike length (if $>1 \mu \mathrm{m}$ ) & $6.89 \pm 0.42 \mu \mathrm{m}(81)$ \\
\hline \multicolumn{2}{|l|}{ Velocity of extension } \\
\hline of microspikes & $4.47 \pm 0.52 \mu \mathrm{m} / \mathrm{min}(17)$ \\
\hline of lamellipodia & $4.14 \pm 0.63 \mu \mathrm{m} / \mathrm{min}(18)$ \\
\hline \multicolumn{2}{|l|}{ Frequency of formation } \\
\hline of microspikes (per growth cone) & $1.89 \pm 0.21 / \min (8)$ \\
\hline \multicolumn{2}{|l|}{ Retrograde velocity } \\
\hline of microspike base & $1.43 \pm 0.31 \mu \mathrm{m} / \mathrm{min}(9)$ \\
\hline of microspike tip & $3.56 \pm 0.68 \mu \mathrm{m} / \mathrm{min}(9)$ \\
\hline Microspike lifespan & $4.49 \pm 0.22 \min (413)$ \\
\hline
\end{tabular}

$1 \mathrm{mg} / \mathrm{ml}$ in $0.15 \mathrm{M}$ borate buffer, $\mathrm{pH}$ 8.3). The coverslips were incubated in this solution at room temperature overnight, washed in HBSS, and then seeded with cells as described above. In this case culture medium lacking serum was used.

Microscopy. Cells were mounted for observation in a chamber fabricated from a $7 \times 4 \mathrm{~cm}$ glass slide in which a 2-cm-diameter circular hole had been drilled. One side of the hole was covered by a $32 \times 32 \mathrm{~mm}$ no. 2 coverslip held in place by silicone grease. The coverslip bearing the cells was inverted onto this assembly and sealed in place by a molten mixture of beeswax, paraffin wax, and petroleum jelly (1:1:1). Slides were then mounted on the stage of an inverted microscope and maintained at $37^{\circ} \mathrm{C}$ by a thermostatically controlled electric fan heater. Observations were made using a phase contrast $\times 100$ planapochromatic phase objective and recorded on Kodak Technical Pan film at ASA 100 using exposures of 6 to $8 \mathrm{sec}$.

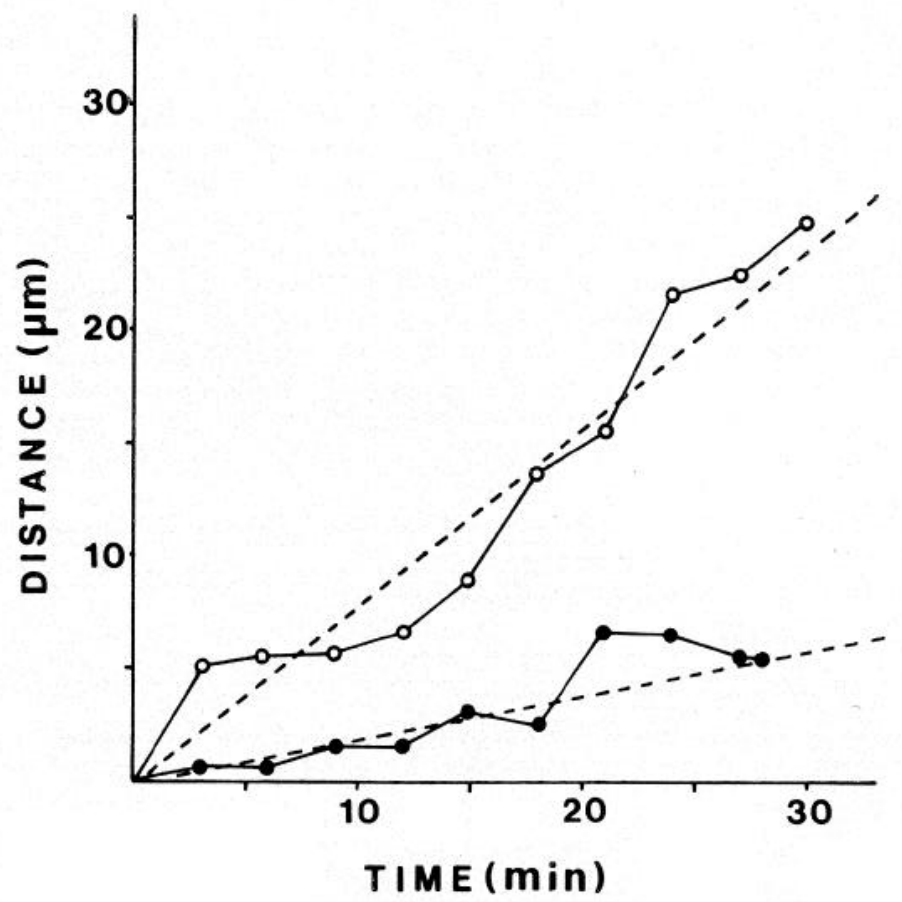

Figure 2. Time course of axonal extension. The vectorial elongation of the axon as distinct from its lateral movements, was measured as described by Katz et al. (1984). O, growth cone on plain glass; $\bullet$, growth cone on polylysine-treated glass. Dashed lines indicate least squares lines of regression.

Growth cones were selected for observation that were actively growing, well isolated from other cells, and possessed a moderate number (about 10) of distinct microspikes. This selection procedure was necessary to carry out our analyses but undoubtedly introduced a bias in favor of smaller, simpler growth cones. Distinct microspikes-those extending more than $1 \mu \mathrm{m}$ from the margin of the cell-were identified and then followed, together with any 


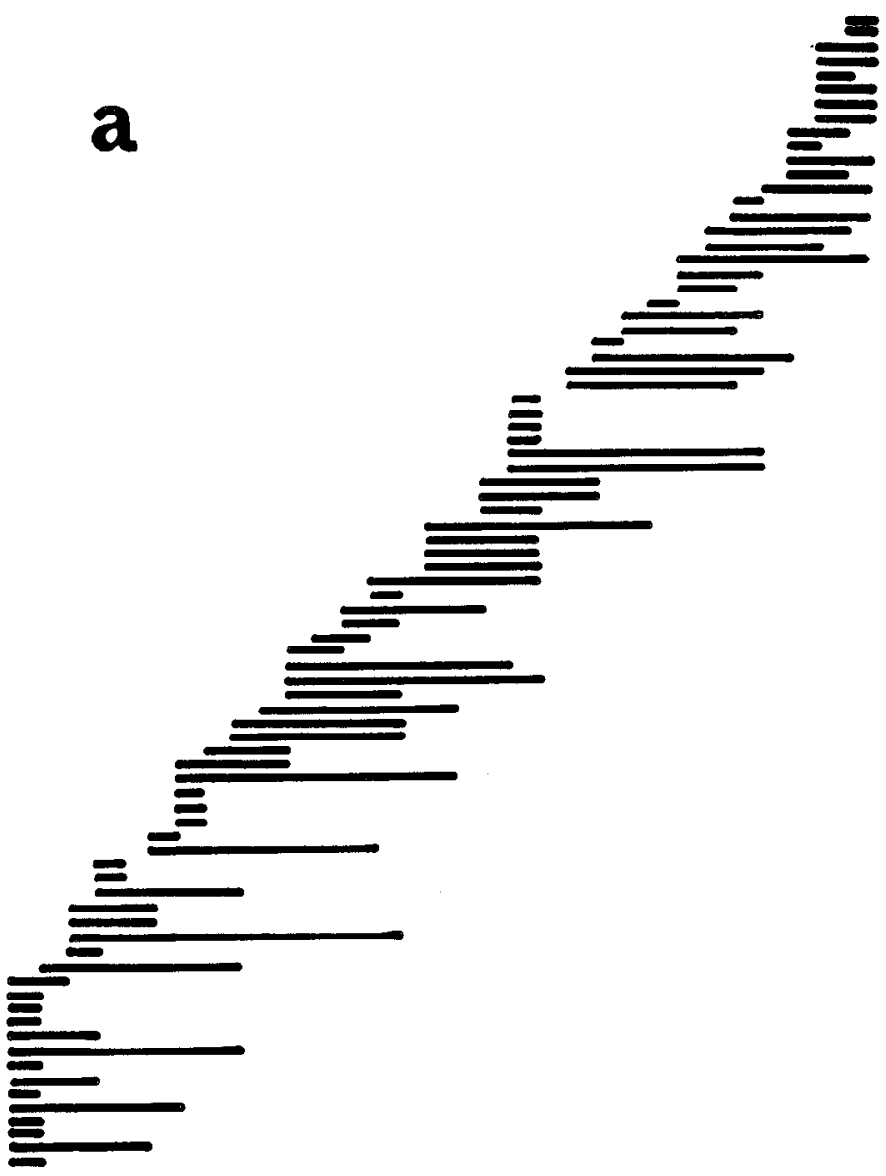

$\begin{array}{llllllllllllllll}1 & 1 & 1 & 1 & 1 & 1 & 1 & 1 & 1 & 1 & 1 & 1 & 1 & 1 & 1 & 1\end{array}$

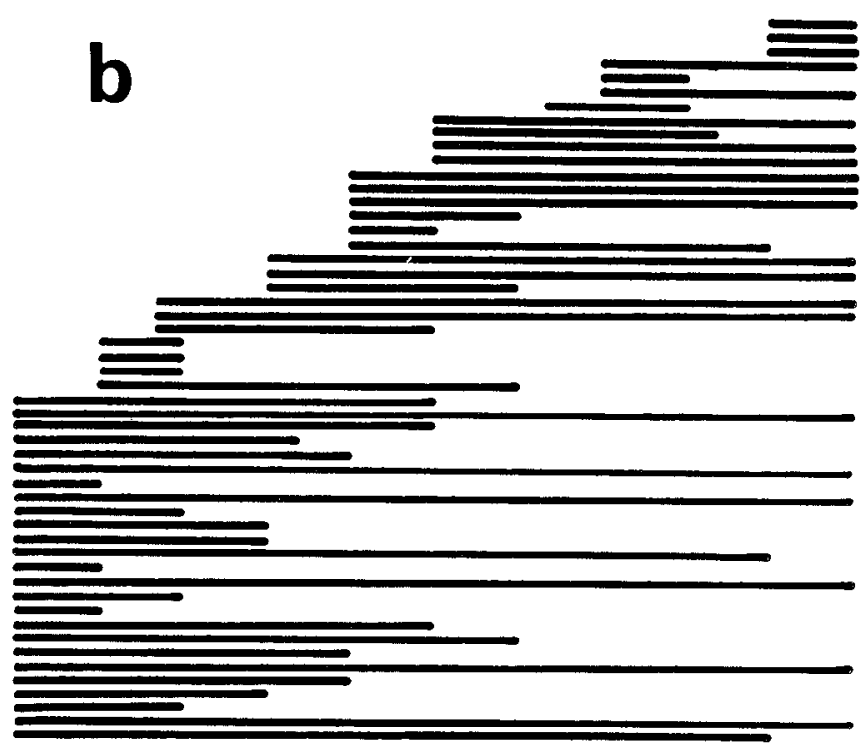

$\begin{array}{lllllcccccccccc}1 & 1 & 1 & 1 & 1 & 1 & 1 & 1 & 1 & 1 & 1 & 1 & 1 & 1 & 1 \\ 0 & & & & 10 & & & 20 & & & 30\end{array}$ microspikes subsequently formed during a period of approximately 30 min. Photographs taken at intervals of $1 \mathrm{~min}$ were collated with records of movement in the intervening periods made by direct observation. This gave a picture of the life cycle of each microspike and, in particular, of their position and mode of formation and loss.

Measurement of growth cone dimensions. Prints of growth cones to a final magnification of $\times 2000$ were prepared for measurement. A point was defined on each micrograph (point $A$ in Fig. 1a) as the intersection of the long axis of the axon with the leading margin of the growth cone, excluding microspikes and fine processes. Distances from point A were measured, parallel to the neurite axis, to the base of each of the most proximal microspikes (those at position 100 to the left and the right-see Fig. 6 for the numbering system employed), and the mean of these two values, $L_{1}$ was calculated. The distance between the two microspike bases at right angles to the axonal axis was also measured and given the value $W_{1}$.

In order to estimate the rate of microspike movement, the outline of each growth cone was replaced by two concentric circles (Fig. 1b). By simple geometry, the inner circle, drawn through points $A B B^{\prime}$, has diameter $D=$ $L_{1}+W_{i}^{2} / 4 L_{1}$, and circumference $\pi D$. The arc of this inner circle over which the microspikes move has length $S_{l}$, where

$$
S_{1}=\pi D\left(1-\frac{\sin ^{-1}\left(W_{1} / D\right)}{180}\right)
$$

and $\sin ^{-1}\left(W_{1} / D\right)$ is in degrees.

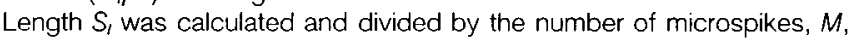
to give the mean distance between the bases of adjacent filopodia, a value used in calculating their rate of movement.

A similar series of calculations for the tips of filopodia used value $W_{f}$ - the distance between the tips of the two most lateral microspikes (Fig. 1a). This was used in place of the diameter $D$ in the above equation to calculate the arc length of the microspike tips $S_{t}$ and the average distance between the tips of adjacent microspikes $\left(S_{f} / M\right)$. Average values of most of these dimensions are given in Table I

\section{Results}

Nine growth cones were photographed at 30 or more intervals of $1 \mathrm{~min}$ as they advanced over plain glass substrata. The photographs were analyzed by the method of Katz et al. (1984) in order to obtain the rates of axonal elongation. In this method, the rate of advance of a growth cone in the direction of the axonal axis is measured separately from lateral movements of the growth cone or changes in its orientation. The average rate obtained in this way for the nine growth cones on plain glass is close to $1.08 \mu \mathrm{m} / \mathrm{min}$ (Fig. 2; see Table I for summary of data for an "average" growth cone under these conditions). Superimposed on this relatively slow progress are the sporadic extension, lateral movement, and retraction of microspikes and lamellipodia, details of which are considered below. In broad terms these movements involve the advance of veils or webs of lamellipodia between adjacent microspikes (see Figs. 10 and 11) and the subsequent extension of microspikes from the lamellipodia base (see Fig. 1), added to an overall retrograde movement of these structures (see Fig. 8).

Filopodial lifespan. Microspikes greater than $1 \mu \mathrm{m}$ in length were identified by letters, as illustrated in Figure 4, and monitored from frame to frame. Over the 30 or so minutes of observation, their total number changes with no obvious relationship to the instantaneous growth rate. The period of time between the appearance of a microspike on a growth cone and its disappearance was recorded, with values ranging from under 2 min to over $30 \mathrm{~min}$ (Fig. 3). The average lifespan for a total of 413 microspikes on nine growth cones, neglecting those present at the beginning or end of the period of measurement, was $4.49 \mathrm{~min}$. This may be compared to the time of $16.8 \mathrm{~min}$ which the average growth cone takes to travel through its own length. Figure 3 also illustrates the longer lifespan of microspikes

Figure 3. Microspike lifespans. Each horizontal line represents an identified microspike. (a) growth cone on plain glass. (b) growth cone on polylysinetreated glass. 
on more adhesive, polylysine-treated surfaces which is discussed below.

Formation of microspikes. Microspikes are produced in several distinct ways. By far the most common is by extension from a lamellipodium, as illustrated in Figure 4. More rarely, microspikes extend from the side of the axon or are produced by the retraction of a lamellipodium. Extension occurs at steady rates, with an average of $4.4 \mu \mathrm{m} / \mathrm{min}$, interspersed with periods of rest or reversal. By
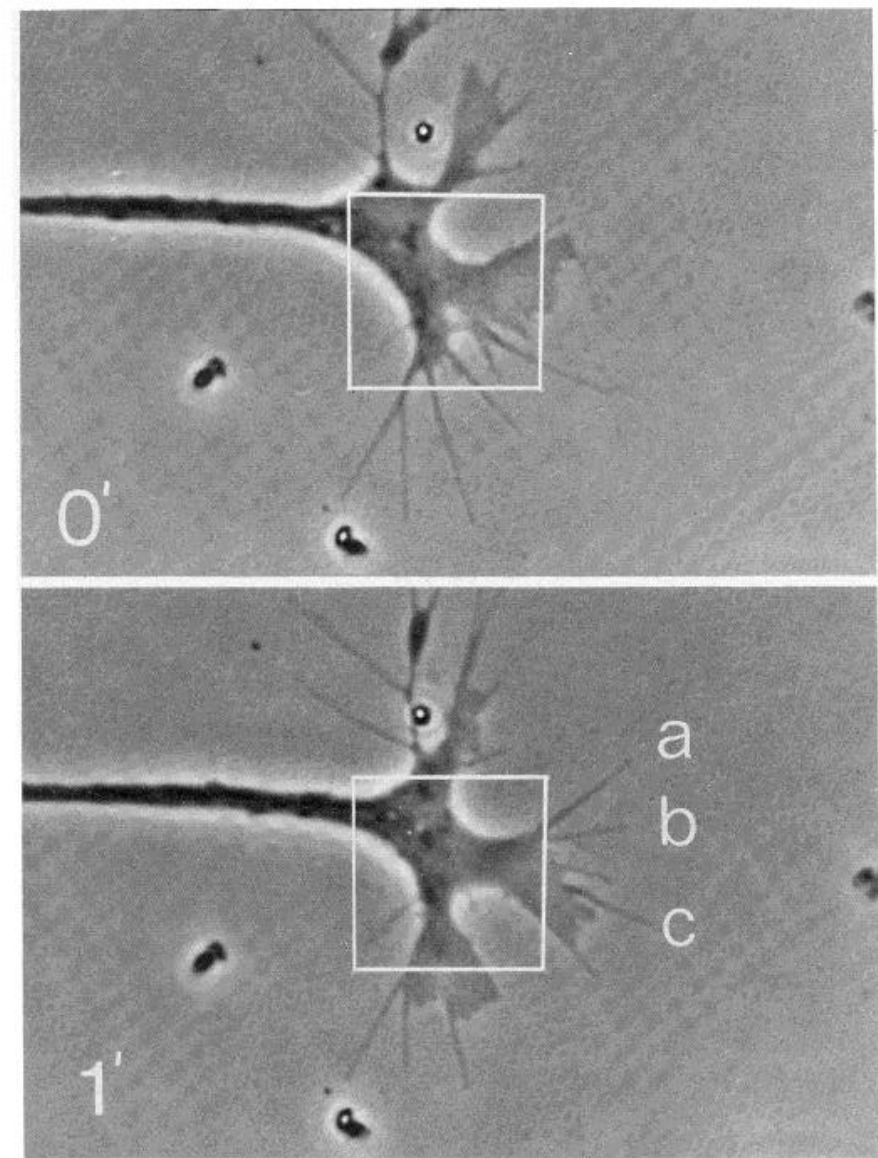

Figure 4. Microspike formation. Microspikes $a, b$, and $c$ are formed at the advancing margin of the lamellipodium. Calibration square $=10 \times 10 \mu \mathrm{m}$, fixed in relation to the substratum.
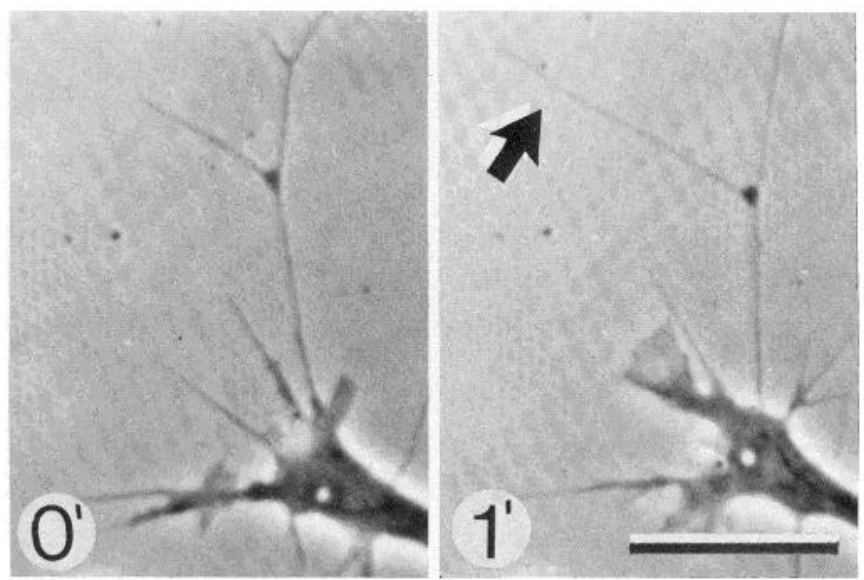

Figure 5. Distal growth of microspikes. A branched lateral microspike increased in length in its distal regions after $1 \mathrm{~min}$ (arrow). Calibration bar, $10 \mu \mathrm{m}$.

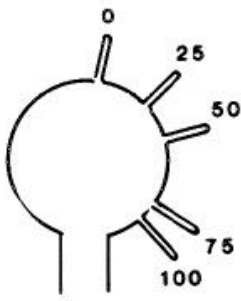

a

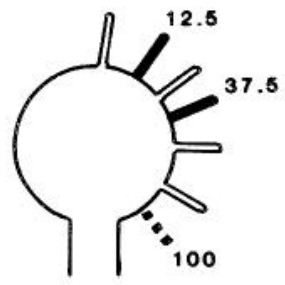

b

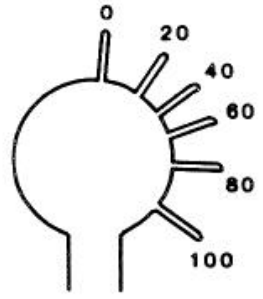

C
Figure 6. Positional values of microspikes. a, Microspikes were assigned equally spaced values between 0 (at the leading apex of the growth cone) and 100 (at the growth cone base), as described in the text. $b$. After an interval of $1 \mathrm{~min}$, the positions of any newly formed microspikes were recorded (shown in the diagram as the solid rectangles at positions 12.5 and 37.5 ) together with the position of any microspikes lost (the dashed line at 100). c. The growth cone microspikes were then renumbered in preparation for the next 60 -sec interval.

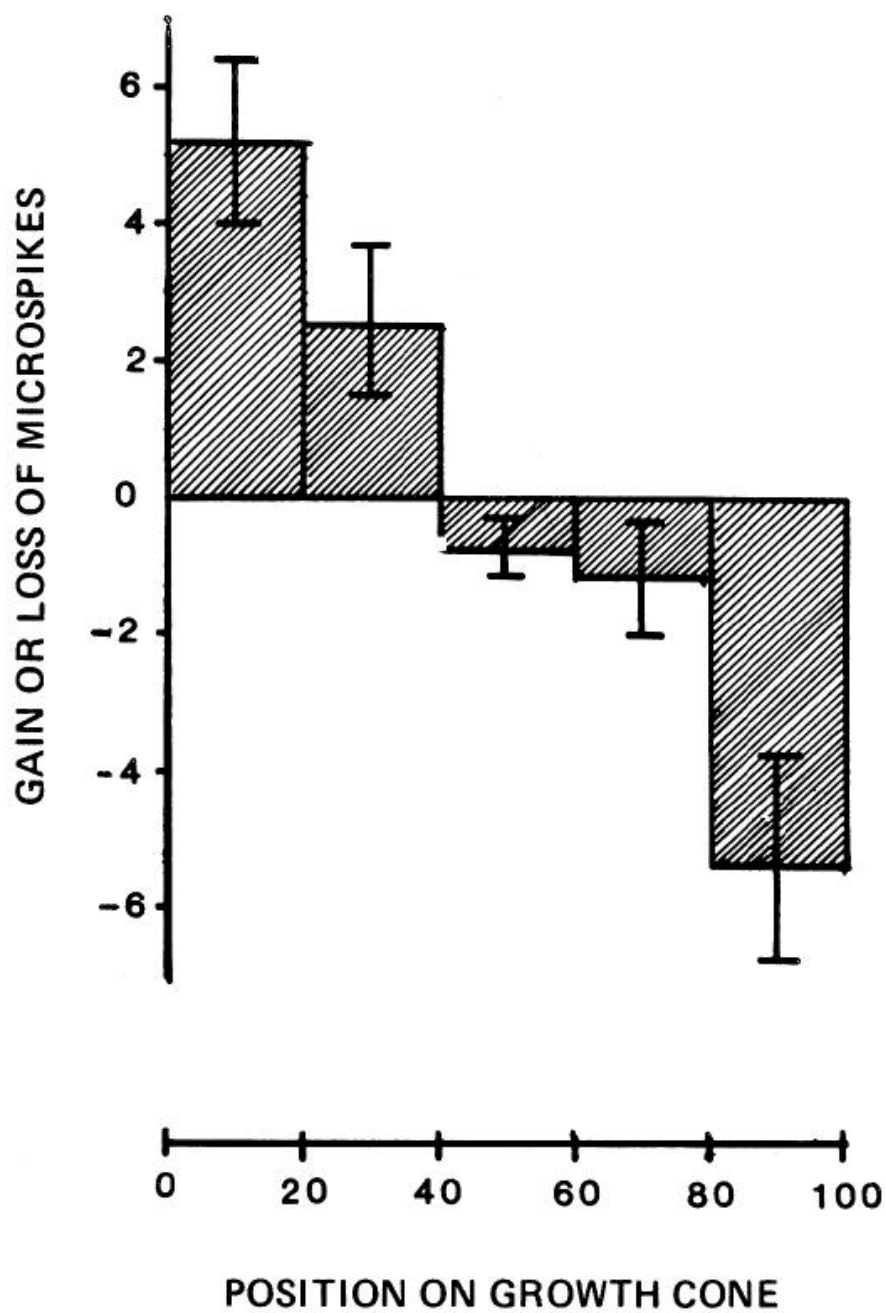

Figure 7. Net gain or loss of microspikes. Each of nine growth cones was observed for 30 to $34 \mathrm{~min}$ at intervals of $1 \mathrm{~min}$. Records of microspike formation and loss were kept and grouped in sectors according to where they occurred on the perimeter of the growth cone: positions ranging from 0 to 100 as illustrated in Figure 6 . In the sector between 0 and 20, for example, a total of 132 microspikes were formed and a total of 85 microspikes were lost with a net gain of 47 microspikes. Since this value was obtained from nine growth cones, the average change in microspike number within this sector is 5.2, as indicated. Error bars indicate the standard error of the mean. 
comparison, in a recent study, Argiro et al. (1985) found that microspikes on the growth cones of rat sympathetic neurons in culture extend at a maximum of rate of $7.2 \mu \mathrm{m} / \mathrm{min}$ shortly after initiation and slow down thereafter. In both the rat and the present chick cultures, instances were recorded in which a bent or branched microspike appeared to elongate at its distal portion (Fig. 5). This may be an indication that the core of the microspikes grows by terminal addition of actin monomers, as suggested for the acrosomal process of Thyone sperm (Tilney and Inoué, 1982).

In order to identify where and when on the growth cone microspikes were formed, an index of position on the growth cone was needed. Changes in form from frame to frame made it difficult to
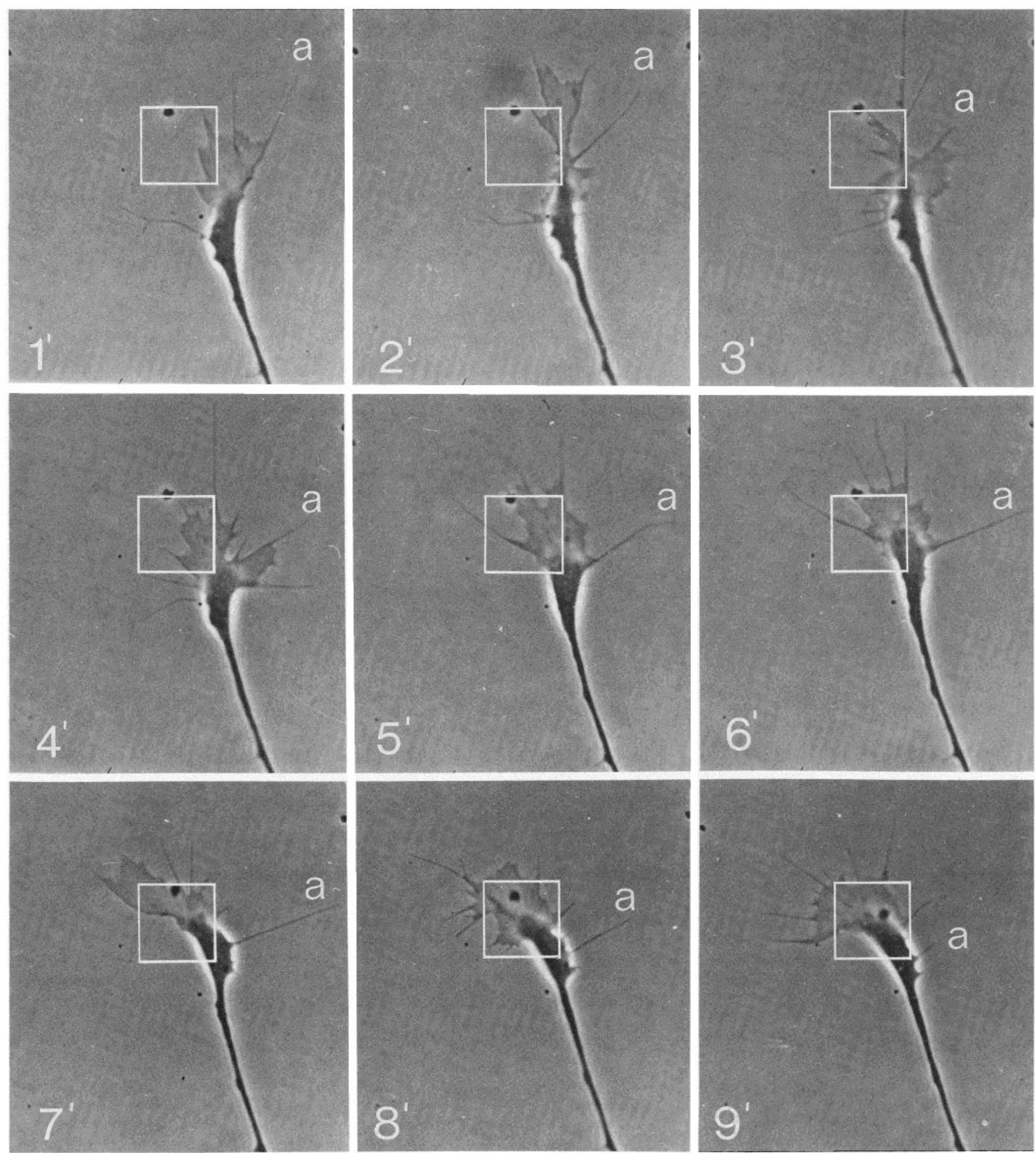

Figure 8. Retrograde movement of microspikes. A newly formed microspike (a) moves backwards from the leading edge of the growth cone to the terminal region of the axon. It also moves in relation to the substratum, as may be seen by reference to the calibration square marking a stationary position on the culture dish. Calibration square $=10 \times 10 \mu \mathrm{m}$. 
assign reference points such as the "centroid" of the growth cone, or the "dividing line" between growth cone and axon. Eventually, after trying several reference systems of this kind, we found that the microspikes themselves made the most reliable markers. They were given positional numbers (in addition to the identifying letters already assigned) which ranged from 0 at the most centrally located microspike at the leading edge of the growth cone to a value of 100 at each of the two most proximal positions on either side of the growth cone. Microspikes at intermediate positions were given values at regular intervals between 0 and 100 , regardless of their precise geographical location: thus, if there were three microspikes between 0 and 100 , these would receive values of 25,50 , and 75 (Fig. 6). Although, for a single growth cone at any instant of time, this system has the disadvantage that numbers do not have precise geometrical meanings, the disadvantage disappears when many sequential images of a growth cone are analyzed together. From the fusion of many images, an average growth cone emerges having a perimeter that is divided into 100 equivalent sectors on either side of the axonal axis.

The above numbering system was used to identify where microspikes were formed and lost. Any microspike lost from the growth cone in going from frame to the next, however this loss occurred, was recorded together with its positional value. Any microspike that appeared was assigned a new positional value halfway between those of its two neighbors and also recorded. At the end of this step, all of the microspikes were given new positional values according to the above-described procedure in preparation for the next time interval (Fig. 6).

Based on an analysis of nine growth cones, each followed for a period of 30 or more minutes, there is a clear difference in the distribution of microspike formation and loss. Formation occurs most frequently at the center of the leading edge (near position 0 ) and decreases toward position 100 at either side. Microspike loss, in contrast, is lowest at the leading edge and increases to a maximum at position 100 on either side. If these values are combined to give

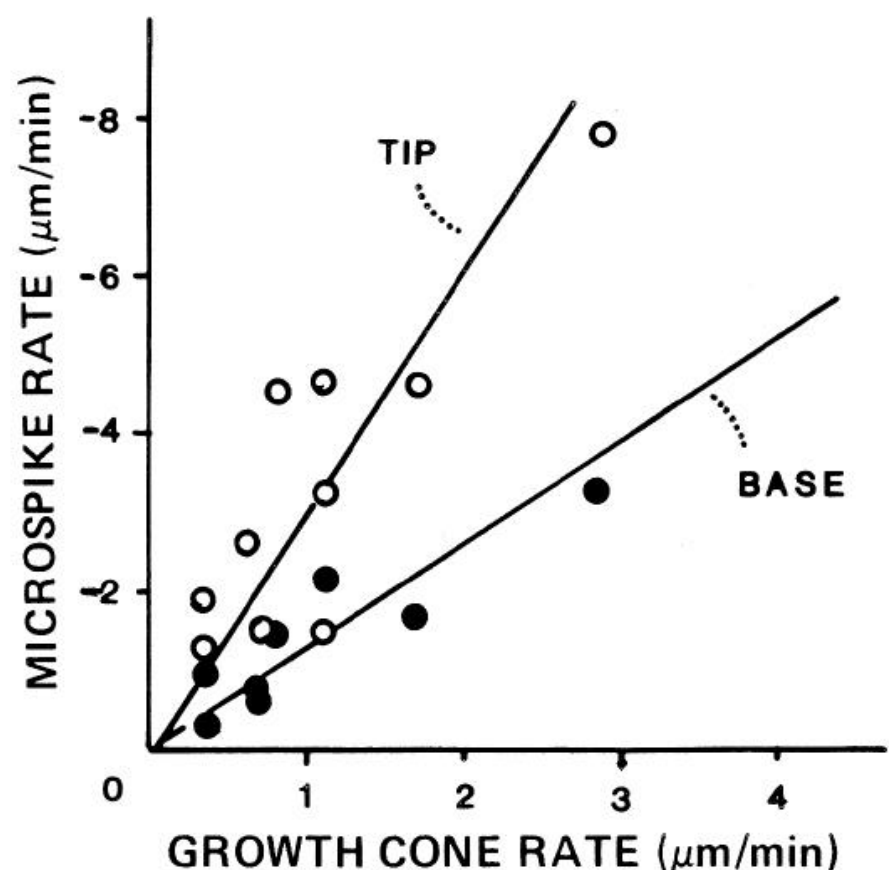

Figure 9. Correlation of microspike movement and growth cone advance. Rates of movement of microspikes at position 50 on the sides of the growth cones were calculated as described in the text. Movement of the bases (O) and tips $(\mathrm{O})$ are plotted separately against growth cone rates together with the least squares lines of regression. Note that microspike movements are negative since they all occur in the retrograde direction.
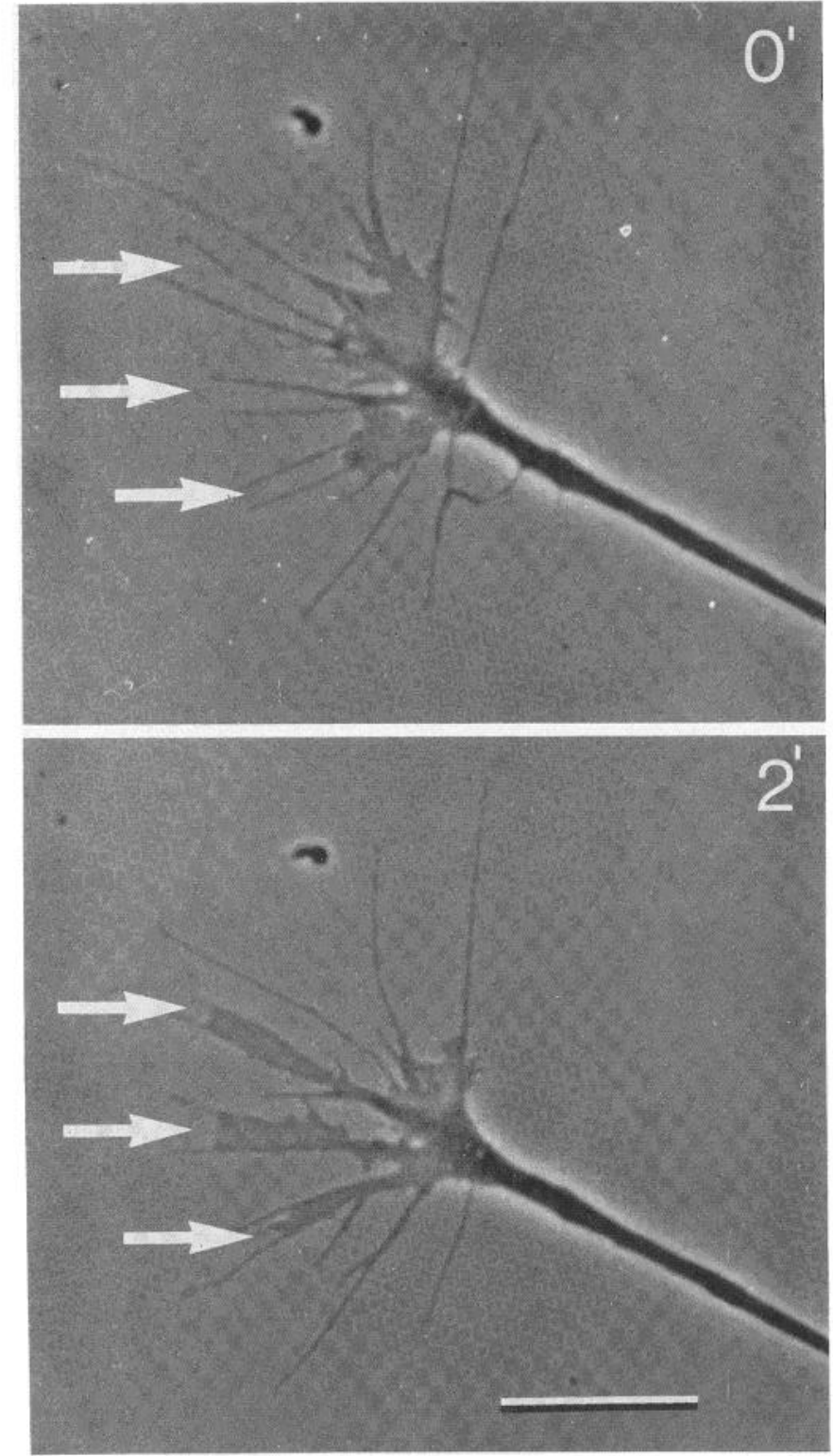

Figure 10. Lamellipodial extension. Regions between adjacent microspikes indicated by arrows at zero time (top) have extended as webs of lamellipodia 2 min later (bottom). Calibration bar, $10 \mu \mathrm{m}$.

the net loss or gain of microspikes at particular positions, they show a progressive change from net addition at position 0 to net loss at position 100 (Fig. 7). It should be noted, incidentally, that this figure combines microspike formation and loss however these occur, including microspike loss by encroachment of lamellipodia or by movements onto the axon. These are discussed separately and in more detail below.

Lateral movements of microspikes. Under the usual tissue culture conditions we employed, the microspikes are capable of moving across the culture substratum. A number of these movements are seen, by comparison to reference marks on the culture surface, to involve the entire length of the microspikes: they are not exclusively anchored at either their tips or their bases (Fig. 8). Whereas most microspikes move in a retrograde direction, others move forward, or not at all.

Since individual microspikes vary a great deal in their movements, it is difficult to estimate their average performance. However, the latter may readily be obtained from the balance of addition and loss of microspikes described in the preceding section. The overall 
number of microspikes does not change in a systematic fashion (see Fig. 3), so that any spatial separation between regions of net gain and loss must be maintained by movement. We should emphasize that the motion referred to here is movement of the position of the microspike on the growth cone. Its motion relative to the substratum will be considered later.

Consider, as an illustration, an idealized growth cone in which microspikes are spaced at equal intervals from position 0 at the apex to position 100 at the base. Now assume that addition occurs only at position 0 , and loss only at position 100 , with these frequencies being equal. Clearly, in order to maintain a steady state, there must be a movement of microspike from position 0 to position 100 , the rate of which will be given by the frequency of microspike gain (or loss) times the average distance between adjacent filopodia.

Microspike movements in a real growth cone can be calculated in a similar fashion. However, in this case, addition and loss are not confined to particular points on the growth cone but are distributed over its entire perimeter. Microspikes consequently move more rapidly at some places than at others; from Figure 7, it can be seen that the net rate of addition of microspikes, summed from position 0 to an intermediate point, $P$, on the growth cone $(S p)$, will reach a maximum at positions close to position 50 and thereafter will decrease to zero. The greatest rate of retrograde movement is, therefore, also a maximum close to position 50 .

Values of $S_{50}$-the net addition or loss of microspikes per minute between positions 0 and 50 , calculated from the data represented in Figure 7-have an average value for nine growth cones of 0.54 \pm 0.11 microspikes/min. Average intermicrospike distances for these nine growth cones, calculated using the method described under "Materials and Methods," give an average value of $0.25 \pm$ $0.57 \mu \mathrm{m}$ from base to base. The average rate of movement at the base of microspikes at position 50 is therefore close to $0.54 \times 2.95$
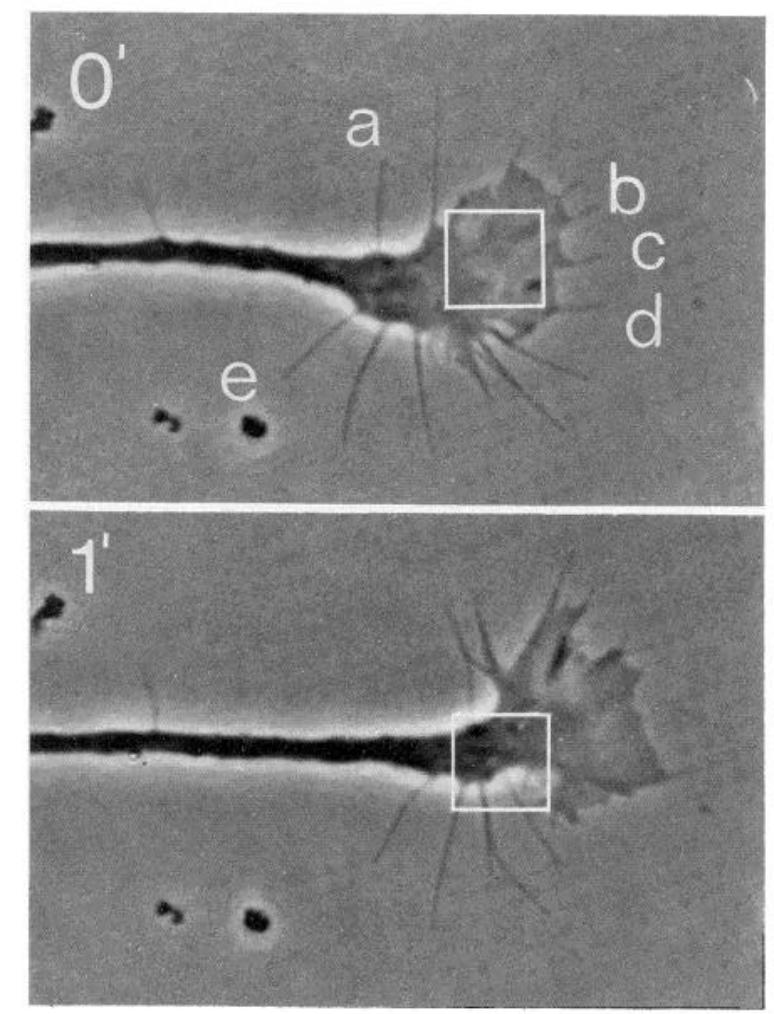

Figure 11. Microspike loss. Micrographs taken at an interval of $1 \mathrm{~min}$ illustrate the two modes of microspike shortening. Microspikes at the base of the growth cone $(a$ and $e$ ) retract into the cell, whereas those at its leading edge $(b, c$, and $d)$ are engulfed by advancing lamellipodial extensions. Calibration square (fixed in relation to the substraum), $10 \times 10 \mu \mathrm{m}$.

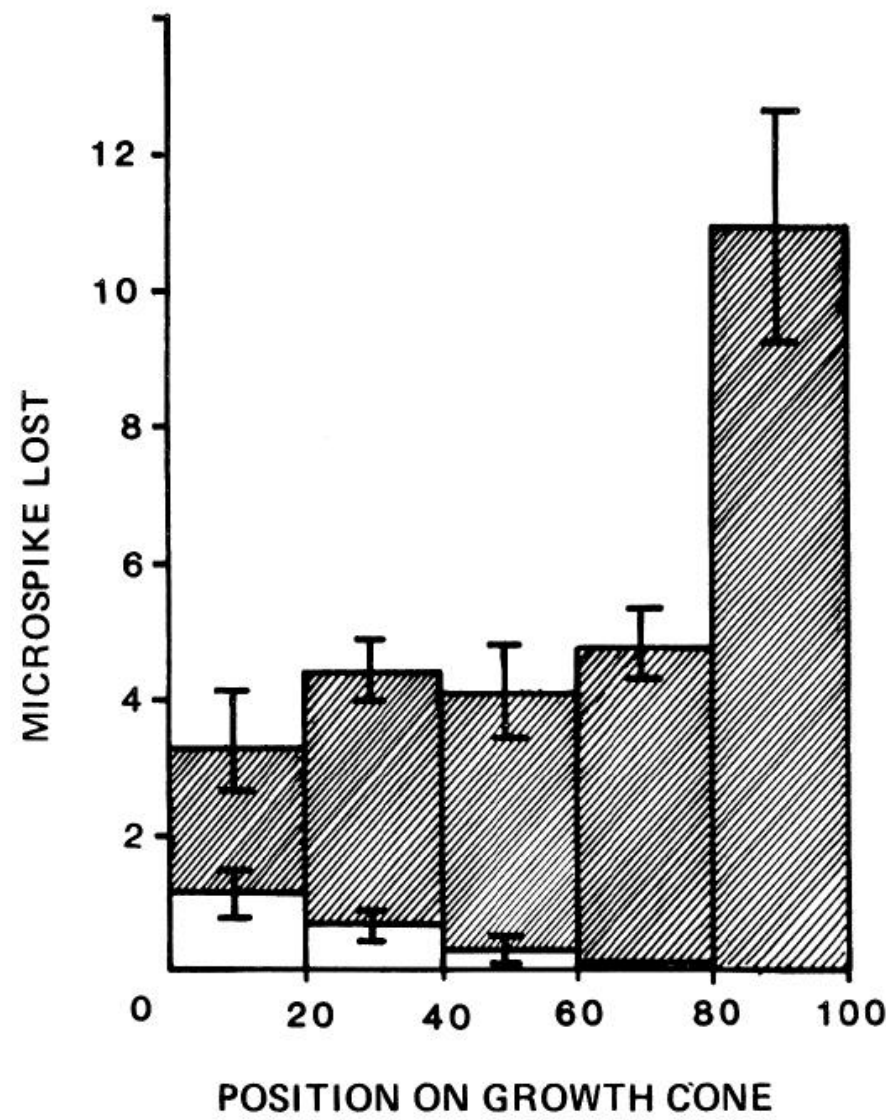

Figure 12. Statistics of microspike loss. The average number of microspikes lost from a growth cone during 30 min of observation is plotted against position on the growth cone (defined as in Fig. 6). Microspikes lost by retraction are indicated by shaded rectangles, those lost by lamellipodial encroachment are indicated by open rectangles. Error bars indicate the standard error of the mean.

$=1.59 \mu \mathrm{m} / \mathrm{min}$ with respect to the growth cone. If rates of microspike movement are calculated separately for each of the nine growth cones, then the average value is $1.43 \pm 0.3 \mu \mathrm{m} / \mathrm{min}$ (Table I), and the rates are found to be correlated with the rate of forward advance of the growth cone (Fig. 9). That is, the bases of the microspikes, on average, remain approximately stationary with respect to the culture substratum as the growth cone moves past them.

If the average distance between the tips of adjacent microspikes, rather than their bases, is used in the above calculation, then the estimated backward movement becomes much greater. Using a tipto-tip distance of $7.57 \pm 0.69 \mu \mathrm{m}$, one obtains a backward movement of $3.57 \mu \mathrm{m} / \mathrm{min}$, which is 3 times faster, although in the opposite direction, than the advance of the growth cone. In relation to the substratum, the microspike tips are moving at an average rearward velocity of $3.57-1.08=2.49 \mu \mathrm{m} / \mathrm{min}$. It should be noted, however, that this assumes that the microspike maintains a radial orientation on the growth cone, whereas those in contact with the substratum are clearly slowed by the adhesive interactions they experience.

Loss of microspikes. The most frequently observed manner of microspike loss was by retraction into the body of the growth cone. This occurred rapidly, usually within a few seconds, and often involved the formation of phase-dense beads at the tip or along the length of a microspike which were subsequently transported back into the cell. Retraction of microspikes showed the same general trend as microspike loss in general; being greatest at the basal positions and least at the leading edge (see Fig. 12). A second method by which microspikes were lost was by engulfment when lamellipodia extended between adjacent microspikes (Fig. 10), and 
beyond the microspike tip (Fig. 11). This event showed the opposite distribution to that of retraction, and was most common at the leading edge and least common at the base (Fig. 12). Clearly this event represents the advance of the leading margin of the growth cone and may be regarded as an alternative form of microspike shortening in which the base, rather than the tip, is moved.

Microspikes at the base of the cone, at position 100 at either side, sometimes moved onto the axon (Figs. 8 and 13). These were included with the microspikes lost from the growth cone for accountancy purposes (Fig. 7) but represented only a small fraction of the total. Of 477 microspikes lost from this position, taken from nine growth cones, 406 were lost by retraction whereas 71 moved onto the axon as just described. Lateral microspikes were also seen, rarely, to form spontaneoúsly by extension from the axon. They then remained fixed in position in relation to the substratum (Fig. 13) until they were eventually lost by retraction.

A number of growth cones were examined growing on more adhesive substrata prepared by treating a carbon-coated glass coverslip with either polylysine or polyornithine. The neurites in these cultures grow more slowly (Fig. 2) than on less adhesive substrata and are crooked rather than straight (Ludueña, 1973). Their growth cones appear to be highly flattened onto the substratum and have longer, more numerous microspikes (Letourneau, 1979), which move less and last longer (Fig. 3). Axons under these conditions also possess large numbers of lateral microspikes (Fig. 14), many of which were observed to be passed on from the growth cone as described above.

\section{Discussion}

Our observations have not included the upper or dorsal surface of the cone, which also carries microspikes and lamellipodia and is

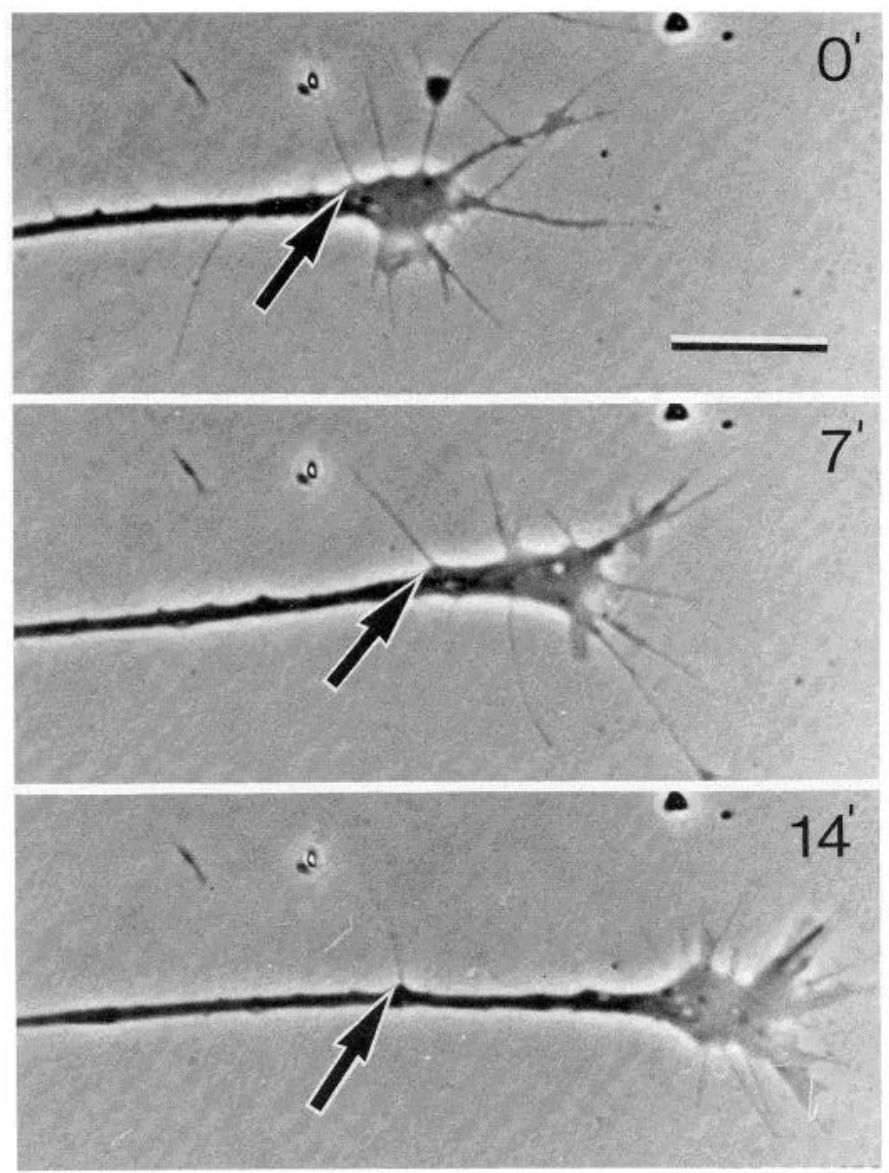

Figure 13. Movement of a microspike from the growth cone onto the axon.

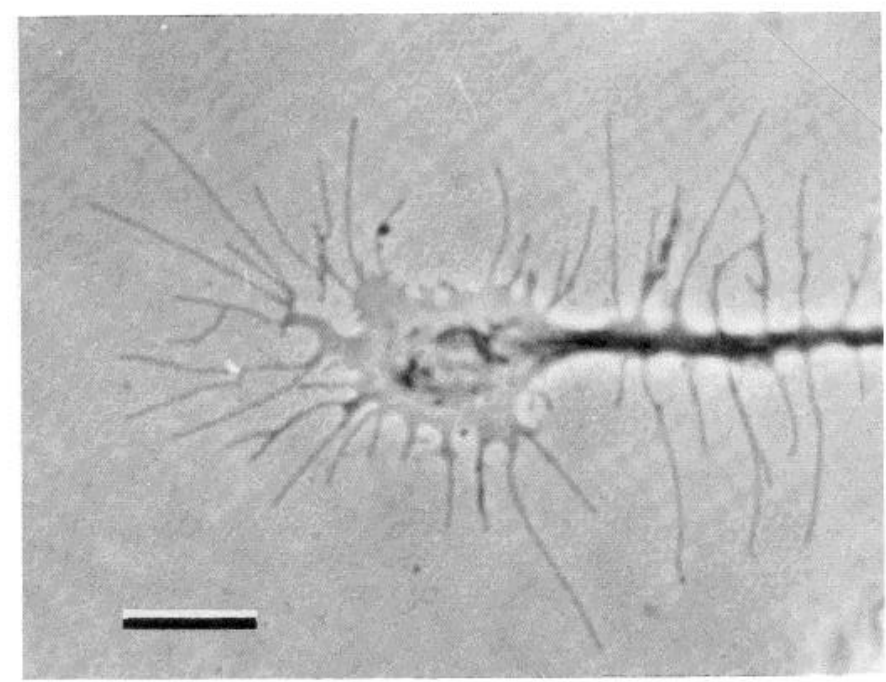

Figure 14. Growth cone on a highly adhesive, polylysine-treated surface Note the large number of lateral microspikes under these conditions. Calibration bar, $10 \mu \mathrm{m}$.

also capable of movement. However, such observations that are available of the dorsal surface indicate that it too exhibits a persistent retrograde movement. Thus, small particles of debris lying on the dorsal surface of the growth cones of cultured sympathetic neurons undergo a retrograde movement as the growth cone advances (Bray, 1970). The retrograde rate reported for this movement (120 $\mu \mathrm{m} / \mathrm{hr}$ ) corresponds closely to the calculated rate of retrograde movement of microspike tips that we have calculated (see Table I).

\section{Summary of microspike behavior}

Where and when are microspikes formed and lost on the growth cone? Microspikes can form or be lost from any position on the perimeter of the growth cone, apparently randomly in time. However, there is a bias toward formation at the leading edge and toward loss at more proximal regions so that microspikes on average move backward in relation to the growth cone. Lamellopodia are produced in close association with microspikes and show a similar regional distribution. In a typical cycle, a lamellipodium extends between two or more adjacent microspikes and then forms the base for the production of new microspikes.

Do microspikes move like limbs or oars that drag the growth cone forward? In a sense, yes: because of the regional differences in the position of formation and loss, the tip of a typical microspike sweeps through an angle of about $65^{\circ}$ in its lifespan of $4 \frac{1}{2} \mathrm{~min}$. It therefore executes a motion somewhat like that of a single stroke of a rowing oar.

However, this analogy is made imperfect by two considerations. First of all, although the tip sweeps backward with respect to the culture substratum, the base of the microspike remains almost stationary. Any forward leverage produced by this sweeping motion of the microspikes would therefore be confined to an inner core of the growth cone (within the inner circle of Fig. $1 b$ ) and will not push the entire growth cone forward. Second, the rowing analogy is imperfect because at the end of its retrograde sweep the microspike shortens while still in contact with the substratum. Unlike the retrieval of an oar from water, the shortening of a microspike is therefore likely to exert a force on the substratum.

From this it seems that "towing" rather than "rowing" may be a more apt simile, with each microspike acting as an inefficiently anchored line, projected ahead of the growth cone and slipping backward over the substratum as tension is applied.

Are the formation and movement of microspikes independent of growth cone advance, indicating a purely sensory role? No. Our observations show that there is a strong correlation between both 


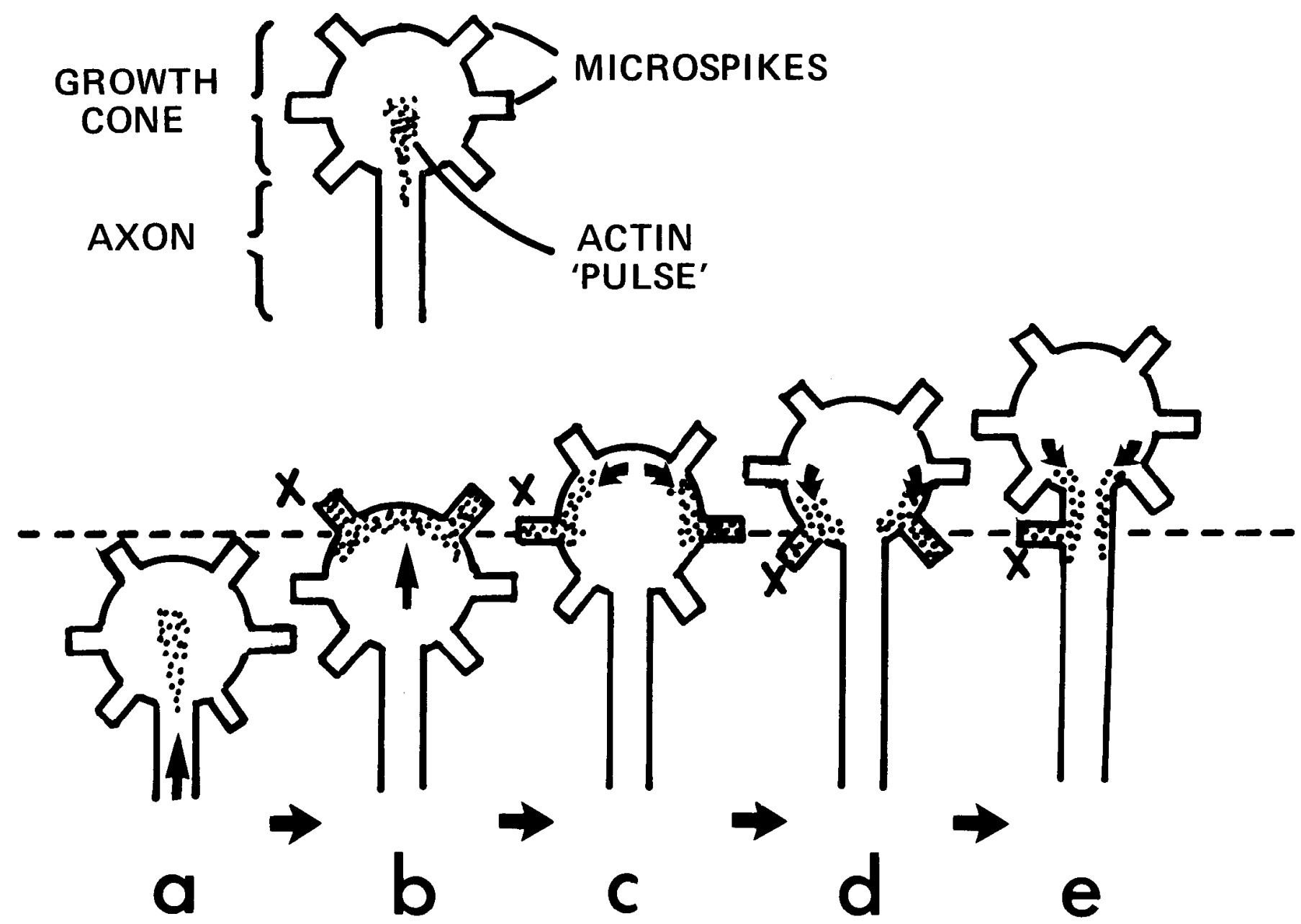

Figure 15. Schematic diagram of the flow of actin-containing structures postulated to occur in the growth cone. The symbols are explained in the upper part of the diagram. As the growth cone advances (a), actin moves from the core of the neurite into the growth cone. Its progress is indicated here by an arbitrary population of actin molecules which are labeled as though in a radioactive "pulse-chase" experiment. In $b$, the actin has added to the cortex and entered into microspikes and other surface protrusions. In $c$ and $d$, the labeled actin cortex moves backwards with respect to the rest of the cone and individual microspikes show some backward movement on the culturc substratum. In $e$, the labeled cortex has been deposited into the neurite. The course of the movements is also shown by an individual microspike, $x$, which eventually adopts a lateral position on the axon.

the rate of formation of microspikes and their retrograde movement and the forward advance of the growth cone. The sensory function of growth cone microspikes impuled by other studies must be closely related to their involvement in locomotion.

Can microspikes move after they have attached and if so are they most firmly anchored at the tip or base? Under the conditions we have used, microspikes do not adhere permanently to the substratum. From the sweeping motion of microspikes in contact with the substratum we have the impression that they have a uniform weak adhesion along their entire length rather than at focal points of adhesion at the tip or base.

What happens to microspikes as the growth cone moves past? How are lateral microspikes formed? The occasional microspike that is not lost while it is on the growth cone remains in position as a lateral microspike on the axon.

How are the microspikes lost and does this play any part in the growth cone advance? At the end of their "life cycle" the growth cone microspikes shorten in length. In the lateral, more proximal, regions, this shortening draws the tip of the microspike back to the growth cone, whereas at the leading edge shortening occurs by the advance of the base. Since in other situations the shortening of microspikes has been shown to produce a contractile force (Nakai, 1960; Albrecht-Buehler, 1976), it seems reasonable to suppose that, in the advancing margin of the growth cone, the shortening of microspikes draws the growth cone forward.

\section{Microspike movements indicate a flow of actin-containing structures}

We have seen that growth cone locomotion is associated with a continual rearward flow of microspikes and associated structures from the leading edge to the base. Since the principal cytoskeletal structures in this region of the cell are actin filaments (Shaw et al., 1981; Letourneau and Ressler, 1983; Tosney and Wessells, 1983) we conclude that a rearward flow of actin filaments also occurs. Suggestions of this kind have been made previously, notably in connection with the migration of fibroblasts in culture (Abercrombie, 1980). The retrograde movement of cross-linked receptors on the dorsal surface of the migrating fibroblasl, for example, is coordinated with the rearward movement of lateral bundles, or "arcs," of actin in its cytoplasm (Heath, 1983).

If actin-rich structures are moving backwards from the apex of the cone, what happens at its base? We suggest that they are there, at least in part, deposited into the relatively stable cortex of the axon. In support of this, microspikes-which have an actin core and presumably are associated with the other regions of the cell cortexoccasionally pass from the growth cone to the axon where they are left behind as the growth cone advances. We also refer to earlier studies in which particles on the surface of axons in culture (Bray, 1970), and branch points in these axons (Bray, 1973), were observed to be left behind as the growth cone advanced, their lack of 
movement being interpreted as evidence for a terminal insertion of plasma membrane. This behavior now seems more likely to be driven by the movements of a network of membrane-associated actin filaments, since this is now realized to be responsible for most of the mechanical properties of the cell surface (Stossel et al., 1982), although it no doubt carries plasma membrane with it. We propose, therefore, that the movements of the growth cone are generated by a "fountain flow" of actin-containing structures including microspikes and lamellipodia as shown in Figure 15. These enter the growth cone from the axon, insert into the apex of the growth cone, move backwards to its base, and then add to the axonal cortex. The pattern is analogous to that occurring on a much larger scale at the tip of an elongating pseudopod of large freshwater amoebae of the Amoeba proteus type, in which a central flow of endoplasmic actin sol is converted into a cortical layer of actin gel (Taylor and Condeelis, 1979).

\section{Growth cone movements could result from regional variations in membrane deformability}

The variations in microspike behavior from leading edge to base parallel far greater differences in surface activity between the growth cone as a whole and the axon. Not only is the axon less active in producing surface extensions of all kinds, but it also shows greatly decreased endocytosis and exocytosis (Bunge, 1977). In broad terms, these differences may be characterized by saying that the surface of the axon (and to a lesser extent, the basal regions of the growth cone) behaves as though it is less "deformable" or has a higher "surface tension" than the surface of the leading margin of the growth cone. Passive mechanical properties such as surface tension are not often considered in discussions of vertebrate cell behavior, perhaps because they are so difficult to measure. But larger cells such as sea urchin eggs have significant, measurable surface tensions-due largely, it is believed, to the tensile properties of a submembranous cortex of protein filaments. Moreover, it is known that these can vary from one region of the cell surface to another, as in the course of cell division (Hiramoto, 1981). We therefore suggest that the appearance of a smoother, more quiescent surface in going from the apex of the growth cone back to the neurite could be due to a progressive increase of surface tension. Such a change could also, in principle, be responsible for the decreased substratum adhesion of the neurite as compared to the growth cone (Gustafson and Wolpert, 1963), and even for the net retrograde flow of surface-associated cytoskeletal elements from the growth cone apex to its base, as discussed in a recent model for cytokinesis (White and Borisy, 1983).

\section{References}

Abercrombie, M. (1980) The crawling movement of metazoan cells. Proc. R. Soc. Lond. (Biol.) 207: 129-147.

Albrecht-Buehler, G. (1976) Filopodia of spreading 3T3 cells. Do they have a substrate-exploring function? J. Cell Biol. 69: 275-286.

Argiro, V., M. B. Bunge, and M. I. Johnson (1984) Correlation between growth cone form and movement and their dependence on neuronal age. J. Neurosci. 4: 3051-3062.
Argiro, V., M. B. Bunge, and M. I. Johnson (1985) A quantitiative study of filopodial growth cone extension. J. Neurosci. Res. 13: 149-162.

Bray, D. (1970) Surface movements during the growth of single explanted neurons. Proc. Natl. Acad. Sci. U. S. A. 65: 905-910.

Bray, D. (1973) Branching patterns of individual sympathetic neurons in culture. J. Cell Biol. 56: 702-712.

Bunge, M. B. (1977) Initial endocytosis of peroxidase or ferritin by growth cones of cultured nerve cells. J. Neurocytol. 6: 407-439.

Bunge, M. B., M. I. Johnson, and V. J. Argiro (1983) Studies of regenerating nerve fibers and growth cones. In Spinal Cord Reconstruction, C. C. Kao, R. P. Bunge, and P. J. Reier, eds., pp. 99-120, Raven Press, New York.

Collins, F. (1978) Axon initiation by ciliary neurons in culture. Dev. Biol. 65: 50-57.

Goodman, C. S., and M. J. Bastiani (1984) How embryonic nerve cells recognize one another. Sci. Am. 251:50-58.

Gustafson, T., and L. Wolpert (1963) The cellular basis of morphogenesis and sea urchin development. Int. Rev. Cytol. 15: 139-214.

Heath, J. P. (1983) Direct evidence for microfilament-mediated capping of surface receptors on crawling fibroblasts. Nature 302: 532-534.

Hiramoto, Y. (1981) Mechanical properties of dividing cells. In Mitosis/ Cytokinesis, A. M. Zimmerman and A. Forer, eds., pp. 398-418, Academic Press, Inc., New York.

Kater. S., and Letourneau, P. C. (1985) Biology of the Nerve Growth Cone. J. Neurosci. Res. 13: 1-335.

Katz, M. J., E. B. George, and L. J. Gilbert (1984) Axonal elongation as a stochastic walk. Cell Motil. 4: 351-370.

Landis, S. (1983) Neuronal growth cones. Annu. Rev. Physiol. 45: 567-580. Letourneau, P. C. (1979) Cell-substratum adhesion of neurite growth cones and its role in neurite elongation. Exp. Cell Rcs. 124: $127 \cdot 138$.

Letourneau, P. C., and A. H. Ressler (1983) Differences in the organization of actin in the growth cones compared with the neurites of cultured neurons from chick embryos. J. Cell Biol. 97: 963-973.

Ludueña, M. A. (1973) The growth of spinal ganglion neurons in serum-free medium. Dev. Biol. 33: 470-476.

Marsh, L., and P. C. Letourneau (1984) Growth of neurites without tilopodial or lamellipodial activity in the presence of cytochalasin B. J. Cell Biol. 99: 2041-2047.

Nakai, J. (1960) Studies on the mechanism determining the course of nerve fibres in tissue culture. Z. Zellforsch. 51: 427-449.

Roberts, A., and J. S. H. Taylor (1983) A study of the growth cones of developing embryonic sensory neurites. J. Embryol. Exp. Morphol. 75: 31-47.

Shaw, G. M. Osborn, and K. Weber (1981) Arrangement of neurofilaments, microtubules and microfilament-associated proteins in cultured dorsal root ganglia cells. Eur. J. Cell Biol. 24: 20-27.

Stossel, T. P., J. H. Hartwig, H. L. Yin, K. S. Zaner, and O. I. Stendahl (1981) Actin gelation and the structure of cortical cytoplasm. Cold Spring Harbor Symp. Quant. Biol. 46: 569-578.

Iaylor, D. L., and J. S. Condeelis (1979) Cytoplasmic structure and contractility in amoeboid cells. Int. Rev. Cytol. 56: 57-144.

Tilney, L. G., and S. Inoué (1982) The acrosomal reaction of Thyone sperm. II. The kinetics and possible mechanisms of acrosomal process elongation. J. Cell Biol. 93: 820-827.

Tosney, K. W., and N. K. Wessells (1983) Neuronal motility: The ultrastructure of veils and microspikes correlates with their motile activities. J. Cell Sci. 61: 389-411

Wessells, N. K., and R. P. Nuttall (1978) Normal branching, induced branching, and steering of cultured parasympathetic motor neurons. Exp. Cell Res. 115: 111-122.

White, J. G., and G. G. Borisy (1983) On the mechanisms of cytokinesis in animal cells. J. Theor. Biol. 101: 289-316. 